\title{
Separability in terms of a single entanglement witness
}

\author{
Piotr Badziąg, ${ }^{1}$ Paweł Horodecki, ${ }^{2}$ Ryszard Horodecki, ${ }^{3}$ and Remigiusz Augusiak ${ }^{4,2}$ \\ ${ }^{1}$ Alba Nova Fysikum, University of Stockholm, S-106 91, Sweden \\ ${ }^{2}$ Faculty of Applied Physics and Mathematics, Gdańsk University of Technology, 80-952 Gdańsk, Poland \\ ${ }^{3}$ Institute of Theoretical Physics and Astrophysics, University of Gdańsk, 80-952 Gdańsk, Poland \\ ${ }^{4}$ ICFO-Institut de Ciències Fotòniques, 08860 Castelldefels (Barcelona), Spain
}

\begin{abstract}
The separability problem is formulated in terms of a characterization of a single entanglement witness. More specifically, we show that any (in general multipartite) state $\varrho$ is separable if and only if a specially constructed entanglement witness $W_{\varrho}$ is weakly optimal, i.e., its expectation value vanishes on at least one product vector. Interestingly, the witness can always be chosen to be decomposable. Our result changes the conceptual aspect of the separability problem and rises some questions about properties of positive maps.
\end{abstract}

PACS numbers: 03.65.-w

Introduction. One of the fundamental problems in quantum information theory concerns detection and characterization of entanglement. In many instances, questions concerning detection can be successfully addressed via the theory of positive maps. There, separability (i.e., the absence of entanglement) of $\varrho \in B\left(\mathcal{H}_{A} \otimes \mathcal{H}_{B}\right)$ is equivalent to the statement that for all positive maps $\Lambda$ acting on $B\left(\mathcal{H}_{A}\right)$, the operator $\sigma=[\Lambda \otimes \mathrm{id}](\varrho)$, with id denoting the identity map acting on $B\left(\mathcal{H}_{B}\right)$, is positive $[1,2]$. Via Jamiołkowski's isomorphism [3], the latter can be reformulated in terms of physical (Hermitian) operators instead of positive maps [2]. Precisely, the state $\varrho$ is separable if and only if the following non-negativity condition

$$
\langle W\rangle_{\varrho}:=\operatorname{Tr}(W \varrho) \geq 0
$$

is satisfied for every Hermitian operator $W \in B\left(\mathcal{H}_{A} \otimes \mathcal{H}_{B}\right)$ such that (a) $\langle\alpha, \beta|W| \alpha, \beta\rangle \geq 0$ for all product vectors $|\alpha, \beta\rangle \equiv|\alpha\rangle \otimes|\beta\rangle \in \mathcal{H}_{A} \otimes \mathcal{H}_{B}$, and (b) there is an entangled state $\sigma \in B\left(\mathcal{H}_{A} \otimes \mathcal{H}_{B}\right)$ for which $\langle W\rangle_{\sigma}<0$.

The importance of this formulation was first recognized by Terhal [4] (see also Ref. [5]), who coined the term entanglement witness for these operators. Also, Terhal pointed out the possibility of experimental entanglement tests via verification of the condition (1) in a laboratory. Since then entanglement witnesses have become one of the most popular tools for entanglement detection, as they allow to identify entanglement without otherwise difficult to avoid complete state tomography [6] (for nonlinear and other methods of entanglement detection see, e.g., Ref. [7] and also the recent reviews [8, 9]). Owing to this, entanglement witnesses have been a subject of rigorous studies leading to a better understanding of their properties and numerous methods of construction (see, e.g., Refs. [8-11]). More importantly, their impressive experimental implementations have been performed [12].

Despite all the progress, practical characterization of the set of entanglement witnesses, which would provide precise optimization parameters is still eluding the researches. Usually, the parameters can only be estimated with limited accuracy $[5,10]$ and the entanglement witnesses have a structure, which is not easy to handle.

As part of the effort to improve on this unsatisfactory situation, in this paper we simplify the conceptual aspect of
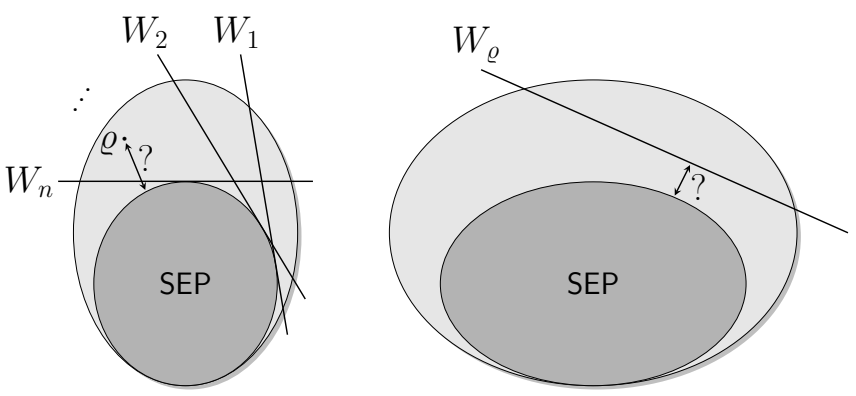

FIG. 1. The separability problem originally expressed in terms of infinitely many entanglement witnesses (left) is here proved to be equivalent to weak optimality of a single entanglement witness in a larger Hilbert space (right).

the separability problem at a cost of the size of the underlying Hilbert space. We consider a given decomposition of a $d_{A} \otimes d_{B}$ state $\varrho \in B\left(\mathcal{H}_{A} \otimes \mathcal{H}_{B}\right)$ and construct an associated entanglement witness $W_{\varrho}$ acting on a larger product Hilbert space $\mathcal{H}^{\prime} \otimes \mathcal{H}^{\prime}$ with $\operatorname{dim} \mathcal{H}^{\prime} \leq\left(d_{A} d_{B}\right)^{4}$. Weak optimality of this witness is then proven to be equivalent to the separability of $\varrho$, where we call a witness weakly optimal if its expectation value vanishes on at least one product vector, or, in other words, it is tangent to the set of separable states (see also Refs. $[10,13]$ for the notion of optimality of the entanglement witnesses).

Our approach has the following conceptual advantage: Since the witness $W_{\varrho}$ can be explicitly calculated, all the elements of the possible subsequent tests have well-defined and clear structures. In particular, the arbitrary multipartite separability problem is here mapped into the analysis of $a$ single bipartite entanglement witness (see Fig. 1). Moreover, our formulation provokes some interesting questions about the structure of the set of the entanglement witnesses and the corresponding maps derived from a given quantum state.

In this context, it is worth noticing that the question of strict positivity of a single entanglement witness on separable states has an algorithmic solution in terms of the so-called Henkel forms. The underlying algorithm was constructed more than three decades ago by Jamiołkowski [14] (see also Ref. [15]). Even though it is not of practical use here, it is still conceptually interesting. In particular, this algorithm can decide the 
witness' weak optimality in a finite, a priori known number of steps.

Construction and the main result. Our state witness $W_{\varrho}$ is constructed from the biconcurrence matrix [16], two forms reflecting its transformation properties and an additional projection. We begin the construction with a decomposition of $\varrho$ in terms of subnormalized vectors, so that $\varrho=\sum_{i}\left|\Psi^{i}\right\rangle\left\langle\Psi^{i}\right|$ (eigendecomposition is usually the most obvious although by no means necessary choice). The decomposition defines the corresponding biconcurrence matrix $B=B(\varrho)$ [16], whose elements can most easily be expressed as [17]:

$B_{m \mu, n \nu}=\left\langle\Psi_{A B}^{m}\left|\left\langle\Psi_{A^{\prime} B^{\prime}}^{\mu}\left|P_{A A^{\prime}}^{\text {asym }} \otimes P_{B B^{\prime}}^{\text {asym }}\right| \Psi_{A B}^{n}\right\rangle\right| \Psi_{A^{\prime} B^{\prime}}^{\nu}\right\rangle$

with $P_{X X^{\prime}}^{\text {asym }}$ being a projector onto the antisymmetric subspace of the Hilbert space $\mathcal{H}_{X} \otimes \mathcal{H}_{X^{\prime}}(X=A, B)$.

The operator $B$ acts on a product Hilbert space $\mathcal{H} \otimes \mathcal{H}$ with the dimension of $\mathcal{H}$ depending on the amount of vectors $\left|\Psi^{i}\right\rangle$ in the above decomposition of $\varrho$. When one begins with the eigendecomposition of $\varrho$, then $\operatorname{dim} \mathcal{H}=d_{A} d_{B}$, which is the maximal number of eigenvectors of $\varrho$. However, in order to allow for a separable decomposition (all vectors $\left|\Psi^{i}\right\rangle$ are product) whenever it exists, one needs $\operatorname{dim} \mathcal{H}=\left(d_{A} d_{B}\right)^{2} \equiv N$ (recall that a separable decomposition of $\varrho$ may require up to $N$ vectors). Consequently, from now on we will regard $B$ as an operator acting on the extended space $\mathcal{H} \otimes \mathcal{H}$ with $\operatorname{dim} \mathcal{H}=N$. One then notices that $B$ is positive and symmetric with respect to the transposition of indices $m$ and $\mu$, as well as $n$ and $\nu$. More importantly, denoting by $P_{\mathrm{cl}}=\sum_{i}|i, i\rangle\langle i, i|$ the classically correlated projector acting on $\mathcal{H} \otimes \mathcal{H}$, it follows from Ref. [16] that $B$ is related to separability of $\varrho$ via the following theorem.

Theorem 1. The state $\varrho \in B\left(\mathcal{H}_{A} \otimes \mathcal{H}_{B}\right)$ is separable if and only if the function

$$
\mathcal{B}(\varrho)=\inf _{U} \operatorname{Tr}\left(P_{\mathrm{cl}} U \otimes U B U^{\dagger} \otimes U^{\dagger}\right),
$$

called biconcurrence function, vanishes. The infimum is taken over all unitary matrices $U$ acting on $\mathcal{H}$.

As each unitary matrix in the above represents an orthonormal basis, we can straightforwardly rewrite (3) as

$$
\mathcal{B}(\varrho)=\inf _{\left\{\left|x_{i}\right\rangle\right\}} \sum_{i=1}^{N}\left\langle x_{i}, x_{i}|B| x_{i}, x_{i}\right\rangle=0,
$$

where the infimum is taken over all orthonormal bases $\left\{\left|x_{i}\right\rangle\right\}$ of $\mathcal{H}$. Consequently, Theorem 1 can be alternatively phrased as follows.

Theorem 2. A bipartite state $\varrho$ is separable if and only if there exists a set of vectors $\left|x_{i}\right\rangle, i=1 \ldots N$, for which the following three forms vanish at the same time:

(i) zero form condition,

$$
G_{0}=\sum_{i=1}^{N}\left\langle x_{i}, x_{i}|B| x_{i}, x_{i}\right\rangle=0
$$

(ii) orthogonality condition for the vectors $\left|x_{i}\right\rangle$,

$$
G_{1}=\sum_{\substack{i, j=1 \\ i \neq j}}^{N}\left|\left\langle x_{i} \mid x_{j}\right\rangle\right|^{2}=0
$$

(iii) normalization condition for the basis $\left\{\left|x_{i}\right\rangle\right\}$,

$$
G_{2}=\sum_{i=1}^{N}\left\|x_{i}\right\|^{4}-\frac{1}{N}\left(\sum_{i=1}^{N}\left\|x_{i}\right\|^{2}\right)^{2}=0 .
$$

Since in general all $G_{0}, G_{1}, G_{2}$ are non-negative, these three conditions can be replaced by a single one: $\alpha G_{0}+\beta G_{1}+$ $\gamma G_{2}=0$ for any fixed $\alpha, \beta, \gamma>0$. In other words, a state $\varrho$ is entangled if and only if the inequality

$$
\alpha G_{0}+\beta G_{1}+\gamma G_{2}>0
$$

holds for any set of vectors $\left\{\left|x_{i}\right\rangle\right\}$ and triple $\alpha, \beta, \gamma>0$.

To convert this into the property (a) of an entanglement witness (see above), we need to extend the Hilbert space once more. Recall that the operator $B$ is defined on $\mathcal{H} \otimes \mathcal{H}$. We extend each $\mathcal{H}$ to $\mathcal{H}^{\prime}=\mathcal{H} \otimes \widetilde{\mathcal{H}}$, where $\widetilde{\mathcal{H}}$ is an auxiliary space isomorphic to $\mathcal{H}$. One then notices that any vector $|u\rangle \in \mathcal{H} \otimes \widetilde{\mathcal{H}}$ can be written in the form

$$
|u\rangle=\sum_{i=1}^{N}\left|x_{i}, i\right\rangle,
$$

where $\left\{\left|x_{i}\right\rangle\right\}$ is a set of arbitrary vectors from $\mathcal{H}$, while $\{|i\rangle\}$ the standard basis in $\widetilde{\mathcal{H}}$. This observation allows us to substitute single vectors in the extended space for the sets of vectors in the conditions (5)-(7). To this end, let us introduce the swap operator $V=\sum_{i j}|i\rangle\langle j|\otimes| j\rangle\langle i|$ that together with $P_{\mathrm{cl}}$ (see above for the definition) will act on $\widetilde{\mathcal{H}} \otimes \widetilde{\mathcal{H}}$. For the sake of clarity, we mark this action by a tilde on top of the relevant operator.

With this notation, we can rewrite the necessary and sufficient condition for entanglement (8) in terms of a degree-four form $A$ as

$$
\mathcal{B}(\varrho)=\min _{|u\rangle \in \mathcal{H} \otimes \widetilde{\mathcal{H}}}\langle u, u|A| u, u\rangle>0 .
$$

The minimum is taken over all vectors $|u\rangle \in \mathcal{H} \otimes \widetilde{\mathcal{H}}$, while the operator $A$ acts on $(\mathcal{H} \otimes \widetilde{\mathcal{H}}) \otimes(\mathcal{H} \otimes \widetilde{\mathcal{H}})$ and reads:

$$
\begin{aligned}
A & =\alpha B \otimes \widetilde{P}_{\mathrm{cl}}+\beta I \otimes\left(\widetilde{V}-\widetilde{P}_{\mathrm{cl}}\right)+\gamma I \otimes\left(\widetilde{P}_{\mathrm{cl}}-\widetilde{I} / N\right) \\
& =\alpha A_{0}+\beta A_{1}+\gamma A_{2} .
\end{aligned}
$$

The parameters $\alpha, \beta, \gamma>0$ here can be chosen at will, and this freedom may be utilized for, e.g., optimization of the numerical separability tests based on condition (10).

Each of the three terms contributing to the operator $A$ has non-negative expectation values on symmetric product vectors $|u, u\rangle$. Neither the whole operator nor any of its parts 
is, however, a witness. On the one hand, the operator $A_{0}$ is positive and clearly weakens the witness obtained using our method (when the constructed witness is so weak that it is not even weakly optimal, then the corresponding state $\varrho$ is entangled). On the other hand, the operators $A_{1}$ and $A_{2}$ do not represent entanglement witnesses since they have negative expectation values on some product vectors $|u, v\rangle$ with $|u\rangle \neq|v\rangle$. One can, nevertheless, remove this disadvantage without affecting the expectation values on symmetric product vectors $|u, u\rangle$ by adding to $A$ a projection on the antisymmetric subspace $P^{\text {asym }}=(1 / 2)(I \otimes \widetilde{I}-V \otimes \widetilde{V})$ with large enough weight. Moreover, without affecting the expectation values $\langle u, u|A| u, u\rangle$ in (10), it is possible to substitute $Y=P^{\mathrm{sym}} A P^{\mathrm{sym}}$ for the original operator $A$. When one has done the latter, then the following lemma gives a straightforward method to calculate a weight with which $P^{\text {asym }}$ has to be added to an operator $A_{1}$ or $A_{2}$ to guarantee its conversion into an entanglement witness.

Lemma 3. Let $X$ be a Hermitian operator acting on a product Hilbert space $\mathcal{H} \otimes \mathcal{H}$ such that $X=P^{\mathrm{sym}} X P^{\mathrm{sym}}$ and $\langle u, u|X| u, u\rangle \geq 0$ for any $|u\rangle \in \mathcal{H}$. Moreover, let $X_{C}=$ $X+C P^{\text {asym }}$, where $P^{\text {asym }}$ projects onto the antisymmetric subspace of $\mathcal{H} \otimes \mathcal{H}$ and $C$ is a real constant. Then the following implications are true:

(i) If $C \geq\|X\|_{\infty}$, then $\left\langle u, v\left|X_{C}\right| u, v\right\rangle \geq 0$ holds for any pair of vectors $|u\rangle,|v\rangle \in \mathcal{H}$,

(ii) if $C \geq 2\|X\|_{\infty}$, then for any pair of vectors $|u\rangle,|v\rangle \in$ $\mathcal{H}$, there exists $|g\rangle \in \mathcal{H}$ such that

$$
\begin{aligned}
\left\langle u, v\left|X_{C}\right| u, v\right\rangle & \geq\left\langle g, g\left|X_{C}\right| g, g\right\rangle \\
& \geq \inf _{|u\rangle \in \mathcal{H}}\langle u, u|X| u, u\rangle(=: \mathcal{X}) .
\end{aligned}
$$

Proof. Taking two arbitrary normalized vectors $|u\rangle,|v\rangle \in \mathcal{H}$, the symmetry $X=P^{\mathrm{sym}} X P^{\mathrm{sym}}$ implies that

$$
\langle u, v|X| u, v\rangle=\langle\Psi|X| \Psi\rangle
$$

where $|\Psi\rangle:=(|u, v\rangle+|v, u\rangle) / 2$. Up to an unimportant global phase factor, the vector $|v\rangle$ can be decomposed as $|v\rangle=$ $a|u\rangle+b\left|u^{\perp}\right\rangle$ with $a, b$ being two non-negative numbers such that $a^{2}+b^{2}=1$ and $\left|u^{\perp}\right\rangle$ denoting a vector orthogonal to $|u\rangle$. Consequently, $|\Psi\rangle=a|u\rangle|u\rangle+b\left(|u\rangle\left|u^{\perp}\right\rangle+\left|u^{\perp}\right\rangle|u\rangle\right) / 2$ and $\|\Psi\|^{2}=a^{2}+b^{2} / 2$. Finally, it is fairly easy to convince oneself that the Schmidt decomposition of $|\Psi\rangle \operatorname{reads}|\Psi\rangle=x|e, e\rangle+$ $y|f, f\rangle$, where $|e\rangle=[1 / \sqrt{2(1+a)}]\left[(1+a)|u\rangle+b\left|u^{\perp}\right\rangle\right]$ and $|f\rangle=[\mathrm{i} / \sqrt{2(1-a)}]\left[(1-a)|u\rangle-b\left|u^{\perp}\right\rangle\right]$ are orthonormal vectors, while $x=(1+a) / 2$ and $y=(1-a) / 2$. All this allows us to write that

$$
\begin{aligned}
\langle u, v|X| u, v\rangle= & \langle\Psi|X| \Psi\rangle \\
= & x^{2}\langle e, e|X| e, e\rangle+y^{2}\langle f, f|X| f, f\rangle \\
& +2 x y \operatorname{Re}(\langle e, e|X| f, f\rangle) \\
\geq & x^{2}\langle e, e|X| e, e\rangle+y^{2}\langle f, f|X| f, f\rangle \\
& -2 x y|\langle e, e|X| f, f\rangle| \\
\geq & x^{2}\langle e, e|X| e, e\rangle+y^{2}\langle f, f|X| f, f\rangle-2 x y C \\
\geq & -\frac{1}{2}\left(1-a^{2}\right) C=-\frac{1}{2}\left(1-|\langle u \mid v\rangle|^{2}\right) C \\
= & -C\left\langle u, v\left|P^{\operatorname{asym}}\right| u, v\right\rangle
\end{aligned}
$$

where the first inequality follows from the fact that $\operatorname{Re} z \leq|z|$ holds for any $z \in \mathbb{C}$, while the second and the third ones from the assumptions that, respectively, $C \geq\|X\|_{\infty}$ and $\langle u, u|X| u, u\rangle \geq 0$ for any $|u\rangle$.

Comparison of the first and the last expression in (14) immediately gives $\left\langle u, v\left|X+C P^{\text {asym }}\right| u, v\right\rangle \geq 0$ for all $|u\rangle,|v\rangle \in$ $\mathcal{H}$ and $C \geq\|X\|_{\infty}$, proving (i).

In order to prove (ii), we can exploit the second inequality in (14). Its right-hand side does not exceed $\left(x^{2}+\right.$ $\left.y^{2}\right)\langle\tilde{e}, \tilde{e}|X| \tilde{e}, \tilde{e}\rangle-2 x y C$, where $|\tilde{e}\rangle=|e\rangle$ if $\langle e, e|X| e, e\rangle \leq$ $\langle f, f|X| f, f\rangle$, and $|\tilde{e}\rangle=|f\rangle$ otherwise. Consequently,

$$
\langle u, v|X| u, v\rangle \geq\left(x^{2}+y^{2}\right)\langle\tilde{e}, \tilde{e}|X| \tilde{e}, \tilde{e}\rangle-2 x y C
$$

which can be rewritten as

$$
\langle u, v|X| u, v\rangle+4 x y C \geq\left(x^{2}+y^{2}\right)\langle\tilde{e}, \tilde{e}|X| \tilde{e}, \tilde{e}\rangle+2 x y C .
$$

Utilizing further the fact that $C \geq\langle\tilde{e}, \tilde{e}|X| \tilde{e}, \tilde{e}\rangle$ on the righthand side of (16), we arrive at

$$
\langle u, v|X| u, v\rangle+4 x y C \geq(x+y)^{2}\langle\tilde{e}, \tilde{e}|X| \tilde{e}, \tilde{e}\rangle,
$$

which, due to the facts that $x+y=1$ and $4 x y=$ $2\left\langle u, v\left|P^{\text {asym }}\right| u, v\right\rangle$, simplifies to

$$
\left\langle u, v\left|X+2 C P^{\text {asym }}\right| u, v\right\rangle \geq\langle\tilde{e}, \tilde{e}|X| \tilde{e}, \tilde{e}\rangle .
$$

After replacing $2 C$ by $C$ and using the assumption that $C \geq$ $2\|X\|_{\infty}$, this finally gives (12), concluding the proof.

Let us notice that the property (ii) implies in particular that $\mathcal{X}_{C}:=\inf _{|u\rangle,|v\rangle}\left\langle u, v\left|X_{C}\right| u, v\right\rangle=\mathcal{X}$. In other words, if we choose a sufficiently large $C$, then the expectation value of $X_{C}$ in a separable state always upper bounds $\mathcal{X}$.

Our matrix $Y$ satisfies the assumptions of the lemma. Consequently $Y_{C}=Y+C P^{\text {asym }}\left(C \geq 2\|Y\|_{\infty}\right)$ is a good candidate for an entanglement witness. In fact, it is a witness, since it has at least one negative eigenvalue. In this way we have arrived at the main result of the paper.

Theorem 4. A bipartite state $\varrho$ is separable if and only if its corresponding entanglement witness $W_{\varrho}=Y_{C}$ with $C>$ $\|Y\|_{\infty}$ is weakly optimal. Moreover, if $C \geq 2\|Y\|_{\infty}$, then the witness satisfies in addition the condition (12), guaranteeing that $\left\langle u, v\left|W_{\varrho}\right| u, v\right\rangle \geq \mathcal{B}(\varrho)$ for all $|u\rangle,|v\rangle$. 
Proof. First, (i) of Lemma 3 guarantees that for any $C \geq$ $\|Y\|_{\infty}, W_{\varrho}$ is an entanglement witness. Then, it follows from the estimation (14) that if $C>\|Y\|_{\infty},\left\langle u, v\left|W_{\varrho}\right| u, v\right\rangle>0$ for all $|u\rangle \neq|v\rangle$, meaning that the witness $W_{\varrho}=Y_{C}$ can be tangent to the set of separable states only on the symmetric product vectors $|u, u\rangle$. This, in view of Theorem 2 and the discussion that follows, means that the state $\varrho$ is separable if and only if the corresponding witness $W_{\varrho}$ is weakly optimal. It should be noticed that if $W_{\varrho}$ is weakly optimal for some $C>\|Y\|_{\infty}$ then it is weakly optimal for any such $C$. To prove the second part of the theorem one combines (ii) of Lemma 3 and (10).

A simple corollary to this theorem provides a direct link between separable states from $B\left(\mathcal{H}_{A} \otimes \mathcal{H}_{B}\right)$ and weakly optimal entanglement witnesses acting on $\mathcal{H}^{\prime} \otimes \mathcal{H}^{\prime} \cong \mathbb{C}^{N^{2}} \otimes \mathbb{C}^{N^{2}}$ with $N=\left(d_{A} d_{B}\right)^{2}$, namely:

Corollary 5. Every separable state with a pure state product decomposition of length $N$ generates a corresponding weakly optimal entanglement witness from $B\left(\mathbb{C}^{N^{2}} \otimes \mathbb{C}^{N^{2}}\right)$.

Clearly, the strongest entanglement witnesses constructed in this way are those for $A_{0}=0$. Even then, however, the witness construction based on Lemma 3, although universal, does not have to produce the most interesting witnesses. To illustrate this point, we consider the choice $\beta=$ $\gamma=1$ and put $A_{0}=0$. The resulting operator (11) is then $A_{12}=I \otimes \widetilde{V}-(1 / N) I \otimes \widetilde{I}$, while its symmetrization reads $Y=(1 / 2)[I \otimes \widetilde{V}+V \otimes \widetilde{I}-(1 / N)(I \otimes \widetilde{I}+V \otimes \widetilde{V})]$. With a little bit of work, one can easily check that $\|Y\|_{\infty}=$ $(N+1) / N$. According to Lemma 3, one then needs to add $[(N+1) / N] P^{\text {asym }}$ to $Y$, in order to secure its conversion into an entanglement witness $W^{\mathrm{sym}}$. Apparently, this is quite unnecessary. Knowing that for any operator $X \in B\left(\mathbb{C}^{n}\right)$, $\|X\|_{\text {Tr }} \leq \sqrt{n}\|X\|_{\mathrm{HS}}\left(\|\cdot\|_{\mathrm{Tr}}\right.$ and $\|\cdot\|_{\mathrm{HS}}$ stand for, respectively, the trace and the Hilbert-Schmidt norm), one can easily show that without any symmetrization, it is enough to add $(2 / N) P^{\text {asym }}$ to $A_{12}$ in order to convert it into a witness operator $W=I \otimes \widetilde{V}-(1 / N) V \otimes \widetilde{V}$. It follows that $W$ belongs to the class of the so-called decomposable witnesses (see Ref. [10]). Witnesses as $W^{\mathrm{sym}}$ and $W$ may still have zero expectation values on some product vectors $|u v\rangle$ with $|u\rangle \neq|v\rangle$. For that, they do not make any good ground for entanglement identification in $\varrho$. To remedy this disadvantage, it is, however, enough to add $P^{\text {asym }}$ with any positive weight to these witnesses (see the comment after theorem 4). While this will not change their expectation values on symmetric product vectors $|u u\rangle$, the new witnesses (let us denote them by $W_{+}^{\text {sym }}$ and $\left.W_{+}\right)$will become strictly positive on all products $|u v\rangle$ with $|u\rangle \neq|v\rangle$. This is enough to guarantee that after the addition of the contribution from $A_{0}$, the resulting witness will be weakly optimal if and only if the state $\varrho$, from which $A_{0}$ (via $B)$ is derived, is separable [18].

Our method of linking separability of a bipartite state to weak optimality of a single entanglement witness readily generalizes for the states shared by many parties. In the latter case, however, different aspects of separability are described by different matrices $B$ [19]. Thus, one will end up with different corresponding operators $A_{0}$, depending on which aspect of multi-partite entanglement (separability) one would like to test. Nevertheless, the design and structure of the stateindependent contributions to our witness $\left(A_{1}\right.$ and $\left.A_{2}\right)$ as well as condition (10), together with (11), will be exactly as in the bipartite case, irrespectively of the number of parties sharing the tested state $\varrho$. Consequently, the design and the properties of $W_{\varrho}$ for a multi-partite $\varrho$ will be exactly the same as in the bipartite case.

Connection to the theory of positive maps. Via the Jamiołkowski isomorphism, the relation between bipartite states and their "state witnesses" directly translates into a relation between bipartite states and positive but not completely positive maps. In particular, it is easy to see that in the isomorphism, operators, which are not weakly optimal, are mapped onto fully mixing maps. These are the maps which transform any state into a positive matrix of full rank. We then have another immediate corollary to Theorem 4.

Corollary 6. A bipartite state $\varrho$ is entangled if and only if a positive map $\Lambda_{\varrho}$ (it can be chosen to be decomposable) corresponding through the Jamiotkowski isomorphism to the witness $W_{\varrho}$ is fully mixing.

Indeed, the choice of parameters $(\beta=\gamma)$ produces decomposable witnesses and thus decomposable maps.

Conclusion. The separability problem is known to be computationally hard [20]. Nevertheless, analysis of the properties of witnesses $W_{\varrho}$ (and maps $\Lambda_{\varrho}$ ) should be at least in some cases relatively straightforward. One can then hope that our approach not only sheds light on the conceptual aspect of the separability problem, but also may become a starting point for the development of more efficient numerical separability tests. Finally, allowing for $\beta \neq \gamma$ in formula (11) may lead to nondecomposable witnesses and nondecomposable maps. This in turn may lead to some questions about the nature of these witnesses, their possible relation to potential bound entanglement in $\varrho$, or their ability to reveal different geometrical properties of the boundary of the set of separable states. We leave these questions for further research.

Acknowledgements. We thank J. Eisert, M. Demianowicz, L. Ioannou, C. Mora and M. Piani for fruitful discussions. This work is supported by Polish Ministry of Science and Education under Grant No. 1 P03B 09529 and EU project SCALA FP6-2004-IST No. 015714. 
223, 1 (1996).

[3] A. Jamiołkowski, Rep. Math. Phys. 3, 275 (1972).

[4] B. Terhal, Phys. Lett. A 271, 319 (2000).

[5] B. Terhal, Linear Algebra Appl. 323, 61 (2000).

[6] F. G. S. L. Brandão, Phys. Rev. A 72, 022310 (2005); K. M. R. Audenaert and M. B. Plenio, New J. Phys. 8, 266 (2006); J. Eisert, F. G. S. L. Brandão, and K. M. R. Audenaert, ibid. 9, 46 (2007); O. Gühne, M. Reimpell, and R. F. Werner, Phys. Rev. Lett. 98, 110502 (2007); Phys. Rev. A 77, 052317 (2008).

[7] O. Gühne et al., Phys. Rev. Lett. 99, 130504 (2007); J. Samsonowicz, M. Kuś, and M. Lewenstein, Phys. Rev. A 76, 022314 (2007); M. Seevinck and J. Uffink, ibid. 78, 032101 (2008); T. Moroder, O. Gühne, and N. Lütkenhaus, ibid. 78, 032326 (2008); R. Augusiak and J. Stasińska, New J. Phys. 11, 053018 (2009); O. Gühne and M. Seevinck, ibid. 12, 053002 (2010).

[8] R. Horodecki et al., Rev. Mod. Phys. 81, 865 (2009).

[9] O. Gühne and G. Tóth, Phys. Rep. 474, 1 (2009).

[10] M. Lewenstein et al., Phys. Rev. A 62, 052310 (2000).

[11] M. Lewenstein, B. Kraus, P. Horodecki, and J. I. Cirac, Phys. Rev. A 63, 044304 (2001); J. K. Korbicz et al., ibid. 78, 062105 (2008); G. Sarbicki, J. Phys. A 41, 375303 (2008); R. Augusiak, J. Tura, and M. Lewenstein, ibid. 44, 212001 (2011); D. Chruściński and G. Sarbicki, ibid. 45, 115304 (2012); K.-C. Ha and S.-H. Kye, J. Math. Phys. 53, 102204 (2012).

[12] M. Barbieri et. al., Phys. Rev. Lett. 91, 227901 (2003); M.
Bourennane et al., ibid. 92, 087902 (2004); K. J. Resch, P. Walther, and A. Zeilinger, ibid. 94, 070402 (2005); J. Altepeter et al., ibid. 95, 033601 (2005); N. Kiesel et. al., ibid. 95, 210502 (2005); H. Häffner et al., Nature (London) 438, 643 (2005); C.Y. Lu et al., Nat. Phys. 3, 91 (2007).

[13] D. Bruss et al., J. Mod. Opt. 49, 1399 (2002).

[14] A. Jamiołkowski, An Effective Method for Investigation of Positive Endomorphisms on the Set of Positive Definite Operators, Nicolaus Copernicus University (Torun, Poland) Report No. 175, 1972 (unpublished).

[15] G. Dahl, J. M. Leinaas, J. Myrheim, and E. Ovrum, Linear Algebra Appl. 420, 711 (2007); J. Sperling and W. Vogel, Phys. Rev. A 79, 022318 (2009); J. M. Leinaas, J. Myrheim, and P. Ø. Sollid, ibid. 81, 062329 (2010).

[16] P. Badziąg et al., J. Mod. Opt. 49, 1289 (2002).

[17] F. Mintert, M. Kuś, A. Buchleitner, Phys. Rev. Lett. 92, 167902 (2004).

[18] Note that in this case one can apply Lemma 3 to the two parts (witnesses) $\alpha A_{0}$ and $\beta A_{1}+\gamma A_{2}$ (equal to $W$ or $W^{\text {sym }}$, respectively) independently. The only effort required here to get the condition (12) is to calculate the norm of matrix $B$ since the norms of $W, W^{\mathrm{sym}}$ are trivially computable.

[19] F. Mintert, M. Kuś, A. Buchleitner, Phys. Rev. Lett. 95, 260502 (2005).

[20] L. Gurvits, in Proceedings of the Thirty-Fifth Annual ACM Symposium on Theory of Computing (STOCâĂŹO3), San Diego, 2003 (ACM, New York, USA, 2003), pp. 10âĂŞ19. 